\title{
THE TRENDS HIGH-CONTRAST IMAGING SURVEY. I. THREE BENCHMARK M DWARFS ORBITING SOLAR-TYPE STARS
}

\author{
Justin R. Crepp ${ }^{1,2}$, John Asher Johnson ${ }^{1}$, Andrew W. Howard ${ }^{3,4}$, Geoff W. Marcy ${ }^{3}$, Debra A. Fischer ${ }^{5}$, \\ Lynne A. Hillenbrand ${ }^{1}$, Scott M. Yantek ${ }^{1}$, Colleen R. Delaney ${ }^{1}$, Jason T. Wright ${ }^{6,7}$, \\ HowARD T. ISAACSON ${ }^{3}$, AND BENJAMIN T. MONTET ${ }^{1}$ \\ ${ }^{1}$ Department of Astronomy, California Institute of Technology, 1200 East California Boulevard, Pasadena, CA 91125, USA; jcrepp@nd.edu \\ ${ }^{2}$ Department of Physics, University of Notre Dame, 225 Nieuwland Science Hall, Notre Dame, IN 46556, USA \\ ${ }^{3}$ Department of Astronomy, University of California, Berkeley, CA 94720, USA \\ ${ }^{4}$ Institute for Astronomy, 2680 Woodlawn Drive, Honolulu, HI 96822-1839, USA \\ ${ }^{5}$ Department of Physics, Yale University, New Haven, CT 06511, USA \\ ${ }^{6}$ Department of Astronomy and Astrophysics, The Pennsylvania State University, University Park, PA 16802, USA \\ ${ }^{7}$ Center for Exoplanets and Habitable Worlds, The Pennsylvania State University, University Park, PA 16802, USA \\ Received 2012 July 9; accepted 2012 October 10; published 2012 November 20
}

\begin{abstract}
We present initial results from a new high-contrast imaging program dedicated to stars that exhibit long-term Doppler radial velocity accelerations (or "trends"). The goal of the TRENDS (TaRgetting bENchmark-objects with Doppler Spectroscopy) imaging survey is to directly detect and study the companions responsible for accelerating their host star. In this first paper of the series, we report the discovery of low-mass stellar companions orbiting HD 53665, HD 68017, and HD 71881 using NIRC2 adaptive optics (AO) observations at Keck. Follow-up imaging demonstrates association through common proper motion. These comoving companions have red colors with estimated spectral types of K7-M0, M5, and M3-M4, respectively. We determine a firm lower limit to their mass from Doppler and astrometric measurements. In the near future, it will be possible to construct three-dimensional orbits and calculate the dynamical mass of HD 68017 B and possibly HD 71881 B. We already detect astrometric orbital motion of HD $68017 \mathrm{~B}$, which has a projected separation of 13.0 AU. Each companion is amenable to AO-assisted direct spectroscopy. Further, each companion orbits a solar-type star, making it possible to infer metallicity and age from the primary. Such benchmark objects are essential for testing theoretical models of cool dwarf atmospheres.
\end{abstract}

Key words: astrometry - stars: individual (HD 53665, HD 68017, HD 71881) - stars: low-mass - techniques: high angular resolution - techniques: radial velocities

Online-only material: color figure

\section{INTRODUCTION}

It is commonly thought that the radial velocity (RV) method for detecting companions to nearby stars only provides information about bodies with short orbital periods. However, Doppler measurements are quite sensitive to distant objects, because the RV semi-amplitude, $K$, decreases slowly with orbital period, $P$ (or semimajor axis, $a$ ), according to

$$
\begin{gathered}
K \propto m \sin (i) P^{-1 / 3} \\
\propto m \sin (i) a^{-1 / 2},
\end{gathered}
$$

where $m$ is the companion mass and $i$ is the orbit inclination. With $\sim 1-10 \mathrm{~m} \mathrm{~s}^{-1}$ precision, $\mathrm{M}$ dwarfs, brown dwarfs, and super-Jupiters are detectable out to tens of AU; it just takes a long time to complete a full orbit (e.g., Howard et al. 2010). Despite having knowledge of only a fraction of an orbital cycle, RV accelerations (trends) are tremendously useful: they demonstrate conclusively that something initially hidden from view is tugging on the visible star.

Informed target selection is an order-of-magnitude effect for substellar companions. For example, wide-separation $(a \gtrsim$ $10 \mathrm{AU}$ ) brown dwarfs are rare, having been found to orbit only $\approx 3 \%$ of solar-type stars (Metchev \& Hillenbrand 2009). And while companions in the planetary-mass regime are expected to be more common, an upper limit to the frequency of superJovian $\left(m \gtrsim 3 M_{J}\right)$ bodies over a similar semimajor axis range is set at $\approx 20 \%$ for solar-type stars (Nielsen \& Close 2010; Vigan et al. 2012). By observing a sample of intrinsically companion-rich sources, those with clear Doppler accelerations, it is possible to bypass the inefficiencies common to highcontrast programs that nominally select stars based solely on age and proximity to the Sun (Masciadri et al. 2005; Biller et al. 2007; Lafrenière et al. 2007; Leconte et al. 2010; Ehrenreich et al. 2010).

In addition to high observing efficiency, there are significant scientific benefits to combining Doppler measurements with high-contrast imaging. RV and direct astrometry observations ${ }^{8}$ may be used in concert to calculate the companion orbit (all six elements) and dynamical mass (Boden et al. 2006). ${ }^{9}$ For example, Crepp et al. (2012) have measured the mass of the benchmark brown dwarf HR $7672 \mathrm{~B}$ with a fractional uncertainty of only $4 \%$ by monitoring its motion over $\sim 33 \%$ of an orbit cycle. Masses determined independent of photometry and spectroscopy inform theoretical atmospheric models by helping to break degeneracies between the various input parameters, such as mass, radius, age, effective temperature, and

\footnotetext{
8 By direct astrometry we are referring to following the companion along its orbit with direct imaging, measuring the sky-projected separation and position angle relative to the star as a function of time. Indirect astrometry involves measuring the position of the star relative to other (distant) nearby stars or fiducial reference points.

9 See Rodigas et al. (2011) for a discussion regarding mass and orbit constraints for the case of Doppler accelerations combined with direct imaging non-detections.
} 
chemical composition (Barman et al. 2011; Janson et al. 2011). Dynamical masses are likewise important for calibrating thermal evolutionary models, providing a measure of substellar objects' luminosity as they fade with time (Stevenson 1991; Burrows et al. 1997). Further, if the companion orbits a solar-type star, the metallicity and age may be inferred from the primary (Liu et al. 2007; Dupuy et al. 2009; Johnson \& Apps 2009; Biller et al. 2010; Crepp et al. 2012).

With this motivation in mind, we have established an interdisciplinary program that uses a combination of Doppler observations and high-contrast imaging. Specifically, we use years of precise RV measurements to identify promising targets for follow-up high-contrast observations. The goals of our program are as follows:

1. detect companions with mass in the $m \approx 5-500 M_{J}$ range;

2. acquire spectrophotometric measurements across a wide bandpass (YJHKLM);

3. perform follow-up direct astrometric measurements to break the sin $i$ inclination degeneracy resulting from Doppler measurements and calculate dynamical masses;

4. test theoretical atmospheric and evolutionary models in a regime where they currently break down (low temperatures).

Based primarily at Keck Observatory, our RV measurements are obtained using the HIgh Resolution Echelle Spectrometer (HIRES; Vogt et al. 1994) at Keck I. These measurements are often augmented by previous and concurrent observations at Lick Observatory. High-contrast imaging observations are obtained using NIRC2 (PI: Keith Matthews) and the Keck II adaptive optics (AO) system (Wizinowich et al. 2000). We are also expanding this program to include $\mathrm{AO}$ observations with the Project 1640 spectral imager at Palomar (Hinkley et al. 2011) and LMIRCam at the Large Binocular Telescope (Skrutskie et al. 2010; Skemer et al. 2012). Our strategy is to maximize on-sky sensitivity by employing all of the powerful techniques recently developed for high-contrast imaging applications, including coronagraphy (Guyon et al. 2006; Crepp et al. 2010) and aggressive point-spread function subtraction to remove residual scattered starlight from images (Marois et al. 2006; Lafrenière et al. 2007; Crepp et al. 2011; Soummer et al. 2012).

We have found that selecting targets based on the presence of an RV trend is an effective approach, allowing one to take a "shortcut" for finding stars likely to host a low-mass companion amenable to direct imaging detection. In this paper, we report the discovery of three $\mathrm{M}$ dwarf companions orbiting solartype stars. Each companion has red colors and a low luminosity; otherwise, they would be noticed as double-lined spectroscopic binaries in RV data. The companions are amenable to AO-assisted spectroscopy (e.g., Bowler et al. 2010; Pueyo et al. 2012) and represent the first discoveries of our program.

\section{OBSERVATIONS}

\subsection{High-resolution Stellar Spectroscopy}

\subsubsection{Doppler Measurements}

Precise RV data were obtained with HIRES (Vogt et al. 1994) at Keck. We use the iodine cell referencing method to calibrate instrument drift and measure Doppler shifts (Marcy \& Butler 1992; Butler et al. 1996). Observations for HD 53665, HD 68017, and HD 71881 began on 1998 January 25, 1997 January 13, and 1997 December 24, respectively. In each

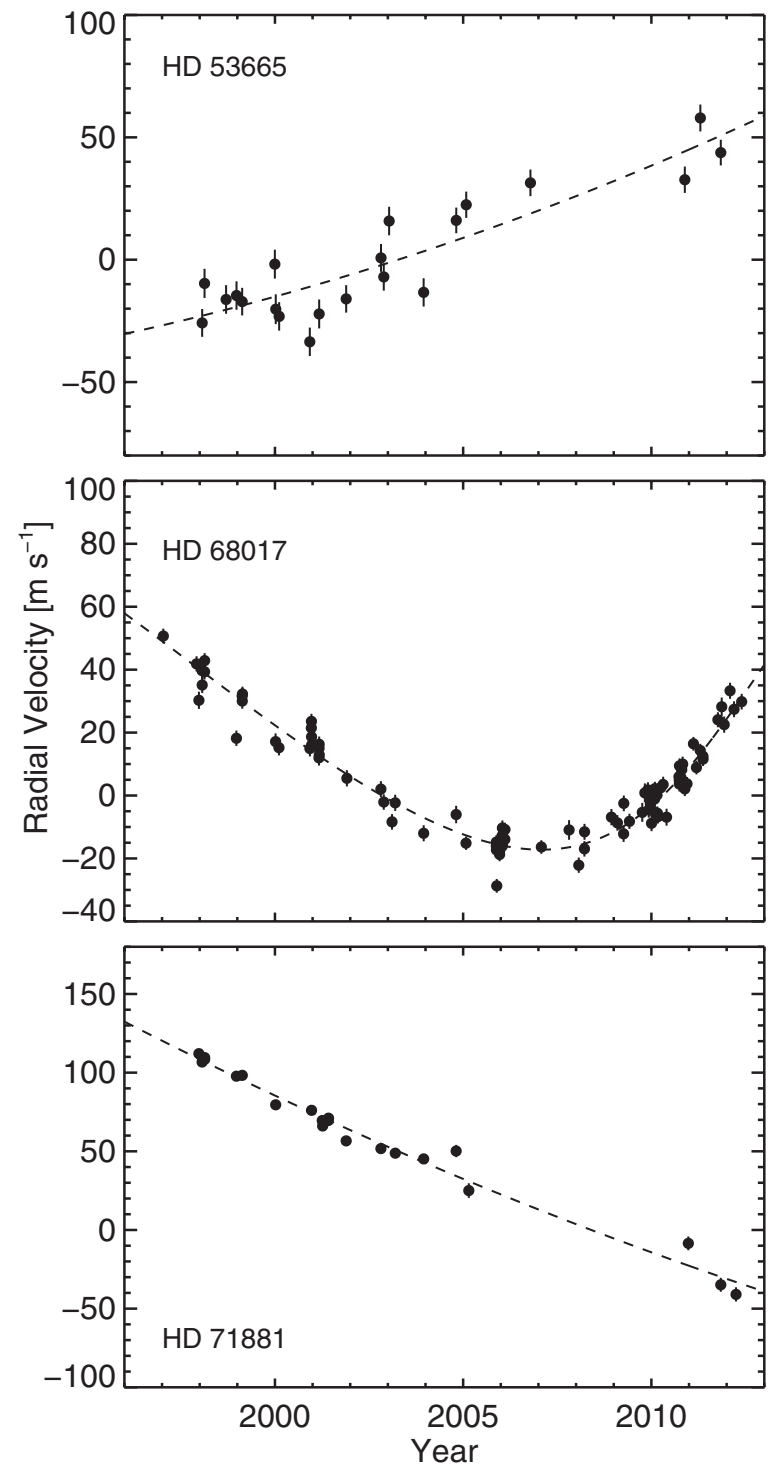

Figure 1. Precise Doppler radial velocity measurements. Our time baseline exceeds a decade for each star. HD 68017 shows significant curvature. It will be possible to calculate the dynamical mass of HD 68017 B with only several more astrometric measurements.

case, a long-term acceleration indicated that the star is orbited by a distant body (Figure 1). Accelerations are approximately linear for HD 53665 and HD 71881, whereas HD 68017 shows significant orbit curvature (change in the acceleration). RV measurements and uncertainties are provided for each star (see Tables 3, 4, and 5) in the Appendix. In Section 4.2, we use Doppler measurements in combination with imaging observations to constrain the mass of each companion.

\subsubsection{Stellar Properties}

Stellar (template) spectra, taken with the iodine gas cell removed from the optical path, were analyzed using the LTE spectral synthesis code Spectroscopy Made Easy (SME) described in Valenti \& Fischer (2005). SME provides an estimate of the stellar effective temperature $\left(T_{\text {eff }}\right)$, surface gravity $(\log g)$, metallicity $([\mathrm{Fe} / \mathrm{H}])$, and projected rotational velocity $(v \sin i)$. Table 2 lists the spectral type and physical properties of each 

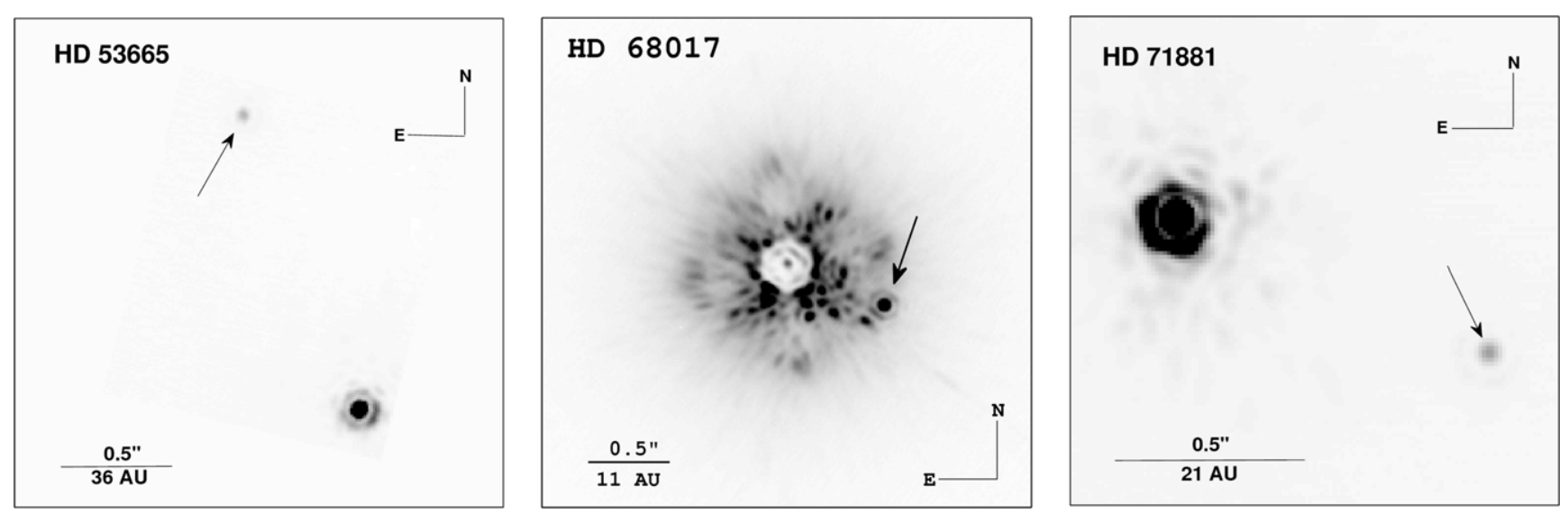

Figure 2. Discovery images of HD 53665 B, HD 68017 B, and HD 71881 B taken in the $K^{\prime}$ filter $\left(\lambda_{c}=2.12 \mu\right.$ m) with NIRC2 on 2011 February 22 . Companions are indicated by an arrow.

Table 1

Summary of Astrometric Measurements

\begin{tabular}{llccrr}
\hline \hline Target Name & \multicolumn{1}{c}{$\begin{array}{c}\text { Date } \\
(\mathrm{UT})\end{array}$} & JD 2,450,000 & \multicolumn{1}{c}{$\begin{array}{c}c \\
(\mathrm{mas})\end{array}$} & \multicolumn{1}{c}{$\begin{array}{c}\text { P.A. } \\
(\mathrm{deg})\end{array}$} & $\begin{array}{c}\text { Proj. Sep. } \\
(\mathrm{AU})\end{array}$ \\
\hline HD 53665 B & 2011 Feb 22 & 5614.79 & $1420.7 \pm 1.0$ & $21.3 \pm 0.1$ & $102.9 \pm 5.5$ \\
& 2012 Jan 7 & 5933.93 & $1413.4 \pm 0.9$ & $20^{\circ} .9 \pm 0.1$ & $102.4 \pm 5.5$ \\
HD 68017 B & 2011 Feb 22 & 5614.81 & $594.5 \pm 0.5$ & $248.2 \pm 0.1$ & $13.0 \pm 0.2$ \\
& 2012 Jan 7 & 5933.96 & $574.6 \pm 0.5$ & $240.3 \pm 0.1$ & $12.5 \pm 0.2$ \\
HD 71881 B & 2011 Feb 22 & 5614.81 & $851.7 \pm 1.1$ & $247.8 \pm 0.1$ & $35.2 \pm 1.0$ \\
& 2012 Jan 7 & 5933.97 & $859.3 \pm 0.6$ & $246.7 \pm 0.1$ & $35.5 \pm 1.0$ \\
\hline
\end{tabular}

star derived from spectral fitting along with comparison to theoretical isochrones.

\subsection{Adaptive Optics Imaging}

HD 53665, HD 68017, and HD 71881 were each discovered on the same night, UT 2011 February 22, using NIRC2 at Keck. We used the narrow camera setting $\left(9.963 \pm 0.006\right.$ mas pixel ${ }^{-1}$ plate scale (Ghez et al. 2008)) to provide fine spatial sampling of the system point-spread function. Images were initially obtained in the $K^{\prime}\left(\lambda_{c}=2.12 \mu \mathrm{m}\right)$ filter for search-mode operation. Companions to each star were noticed in raw frames. We executed a three-point dither pattern to facilitate removal of sky background noise. The companion orbiting HD 68017 is fainter and closer to its host star compared to the other targets, so we also obtained images placing the star behind the (partially transmissive) 300 mas diameter coronagraphic mask.

Images were processed by flat fielding, correcting for hot pixels with interpolation, subtracting the sky background, and rotating the frames to standard northeast orientation. Figure 2 shows fully processed images of each companion. We acquired follow-up observations on UT 2012 January 7 in different filters to obtain colors and assess whether the candidates were associated with their host star. The angular separation and position angle of each companion are listed in Table 1. Brightness ratios are listed in Table 2.

\section{ASTROMETRY}

Our astrometric observations consist of two epochs separated by 0.9 years for each source (Table 2). All three stars have large proper motions (see Table 1), allowing us to easily determine whether the companions share the same space motion over this time frame. We measured an accurate separation and position angle of each companion following the technique described in Crepp et al. (2012). We first fit two-dimensional Gaussian functions to the stellar and companion point-spread functions to locate their centroids in each frame. The primary star was not saturated in any of our dithered images. We then correct for distortion in the NIRC2 focal plane using publicly available solutions provided by Keck Observatory's astrometry support page. ${ }^{10}$ The results are averaged, and uncertainty in the separation and position angle is taken as the standard deviation, taking into account uncertainty in the plate scale and orientation of the array by propagating these errors to the final calculated position.

Figure 3 shows multi-epoch astrometry measurements plotted against the expected motion of a distant background object. We find that all three companions, HD 53665 B, HD $68017 \mathrm{~B}$, and HD $71881 \mathrm{~B}$, are each clearly associated with their respective host stars, implying that they are gravitationally bound. The positions of HD $53665 \mathrm{~B}$ and HD $71881 \mathrm{~B}$ have changed by $\approx 1-2$ pixels over the 0.9 year time frame. Meanwhile, an unrelated background source placed at infinite distance would have moved relative to the host star by 163.7 mas (16.4 pixels) and 296.5 mas (29.8 pixels), respectively. HD $68017 \mathrm{~B}$ has the smallest projected separation of the three $(13.0 \pm 0.2 \mathrm{AU}, 2011$ February) and appears to exhibit significant orbital motion in a clockwise direction.

\section{COMPANION MASS ESTIMATE}

\subsection{Mass from Photometry}

We measured the brightness of each companion relative to its host star by performing aperture photometry, accounting for contamination from the primary. Stellar magnitudes were first converted from Two Micron All Sky Survey (2MASS)

$\overline{10}$ http://www2.keck.hawaii.edu/inst/nirc2/forReDoc/post_observing/dewarp/ 

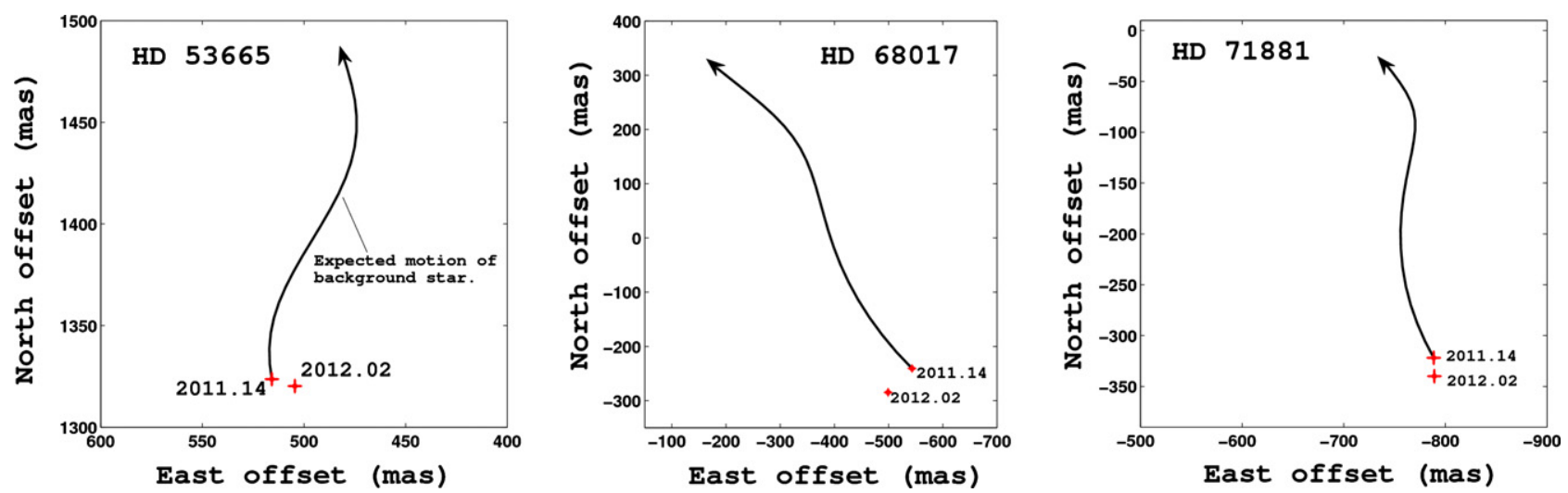

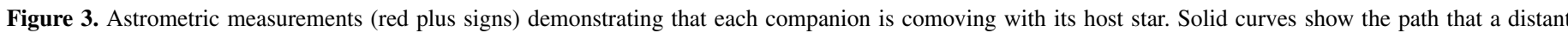
background object would execute over the same time frame accounting for proper motion and parallactic motion from 2011 February 22 through 2012 January 7.

(A color version of this figure is available in the online journal.)

Table 2

System Properties

\begin{tabular}{|c|c|c|c|}
\hline Parameter & HD 53665 A & HD $68017 \mathrm{~A}$ & HD $71881 \mathrm{~A}$ \\
\hline R.A. (J2000) & 070552.8 & 081138.6 & 083155.0 \\
\hline Decl. (J2000) & -010113.7 & +322725.7 & +503700.1 \\
\hline$B$ (mag) & $7.76 \pm 0.02$ & 7.50 & 8.06 \\
\hline$V(\mathrm{mag})$ & $7.26 \pm 0.01$ & 6.81 & 7.43 \\
\hline$J_{2 \mathrm{MASS}}(\mathrm{mag})$ & $6.294 \pm 0.024$ & 5.48 & $6.284 \pm 0.020$ \\
\hline$H_{2 \text { MASS }}(\mathrm{mag})$ & $6.066 \pm 0.027$ & 5.15 & $6.027 \pm 0.027$ \\
\hline$K_{2 \mathrm{MASS}}(\mathrm{mag})$ & $5.983 \pm 0.018$ & 5.09 & $5.959 \pm 0.029$ \\
\hline$d(\mathrm{pc})$ & $72.2_{-4.0}^{+3.9}$ & $21.8_{-0.3}^{+0.2}$ & $41.3_{-1.2}^{+1.1}$ \\
\hline p.m. $\left(\right.$ mas $\left.\mathrm{yr}^{-1}\right)$ & $9.4,-15.1$ & $-462.6,-644.2$ & $-81.4,-338.6$ \\
\hline Spec. type & F8V & G4V & G1V \\
\hline$M_{*}\left(M_{\odot}\right)$ & $1.51_{-0.27}^{+0.20}$ & $0.85_{-0.03}^{+0.04}$ & $1.04_{-0.05}^{+0.06}$ \\
\hline$T_{\text {eff }}(\mathrm{K})$ & $6225 \pm 44$ & $5552 \pm 44$ & $5821 \pm 44$ \\
\hline $\log g\left(\mathrm{~cm} \mathrm{~s}^{-2}\right)$ & $4.05 \pm 0.06$ & $4.65 \pm 0.06$ & $4.29 \pm 0.06$ \\
\hline$[\mathrm{Fe} / \mathrm{H}]$ & $0.17 \pm 0.04$ & $-0.44 \pm 0.03$ & $-0.05 \pm 0.03$ \\
\hline$v \sin i\left(\mathrm{~km} \mathrm{~s}^{-1}\right)$ & $8.6 \pm 0.5$ & $0.8 \pm 0.5$ & $2.1 \pm 0.5$ \\
\hline $\log R_{H K}^{\prime}$ & $-5.022 \pm 0.009$ & $-4.928 \pm 0.013$ & $-5.043 \pm 0.002$ \\
\hline \multirow[t]{2}{*}{ Age (Gyr) } & $2.1_{-0.4}^{+0.8}$ & $4.6_{-1.0}^{+0.9}$ & $4.3_{-1.2}^{+1.0}$ \\
\hline & HD 53665 B & HD $68017 \mathrm{~B}$ & HD $71881 \mathrm{~B}$ \\
\hline$\Delta J_{\mathrm{MKO}}$ & $3.85 \pm 0.15$ & $\cdots$ & $\cdots$ \\
\hline$\Delta H_{\mathrm{MKO}}$ & $3.26 \pm 0.07$ & $>4.16$ & $4.26 \pm 0.04$ \\
\hline$\Delta K_{\mathrm{MKO}} \approx \Delta K_{\mathrm{MKO}}^{\prime}$ & $3.14 \pm 0.10$ & $4.92 \pm 0.10$ & $4.12 \pm 0.08$ \\
\hline$J_{\mathrm{MKO}}$ & $10.12 \pm 0.16$ & $\cdots$ & $\cdots$ \\
\hline$H_{\mathrm{MKO}}$ & $9.31 \pm 0.08$ & $>9.29$ & $10.26 \pm 0.07$ \\
\hline$K_{\mathrm{MKO}}$ & $9.11 \pm 0.11$ & $10.00 \pm 0.11$ & $10.07 \pm 0.09$ \\
\hline$M_{J_{\mathrm{MKO}}}$ & $5.82 \pm 0.20$ & $\cdots$ & $\cdots$ \\
\hline$M_{H_{\mathrm{MKO}}}$ & $5.01 \pm 0.14$ & $>7.60$ & $7.18 \pm 0.09$ \\
\hline$M_{K^{\prime}{ }_{\mathrm{MKO}}}$ & $4.81 \pm 0.16$ & $8.31 \pm 0.11$ & $6.99 \pm 0.11$ \\
\hline Spec. type & K7-M0 & M5 & M3-M4 \\
\hline$m_{\text {dyn }}\left(M_{\odot}\right)$ & $>0.63$ & $>0.08$ & $>0.17$ \\
\hline$m_{\text {model }}\left(M_{\odot}\right)$ & $0.65 \pm 0.03$ & $0.16 \pm 0.02$ & $0.31 \pm 0.03$ \\
\hline$m_{\text {empirical }}\left(M_{\odot}\right)$ & $0.70 \pm 0.03$ & $0.15 \pm 0.01$ & $0.29 \pm 0.02$ \\
\hline
\end{tabular}

Notes. Top: coordinates, apparent magnitude, distance, proper motion (p.m.), spectral type, and physical properties of each host star. Near-infrared magnitudes for the primary star are from 2MASS (Skrutskie et al. 2006). Distance estimates are based on measured parallax from the Hipparcos satellite (van Leeuwen 2007). Effective temperature $\left(T_{\text {eff }}\right)$, surface gravity $(\log g$ ), and metallicity $([\mathrm{Fe} / \mathrm{H}])$ are derived using SME. Ages are estimated using theoretical isochrones for HD 53665 ( $B-V=0.47$ ) (Valenti \& Fischer 2005) and gyrochronology for HD 68017 ( $B-V=0.69$ ) and HD 71881 ( $B-V=0.63$ ) (Mamajek $\&$ Hillenbrand 2008). Bottom: companion magnitude difference, absolute magnitude, estimated spectral type, mass constraint from dynamics $\left(m_{\mathrm{dyn}}\right)$, and estimated mass from photometry using the Dotter et al. (2008) atmospheric models $\left(m_{\text {model }}\right)$ and Delfosse et al. (2000) $M_{K}$-mass empirical relations ( $\left.m_{\text {empirical }}\right)$. measurements (Skrutskie et al. 2006) to the MKO filter system (Tokunaga et al. 2002) using transformations from Carpenter (2001). ${ }^{11}$ Our observations were acquired in the $J, H, K^{\prime}$ (MKO) bands. We have assumed that $\Delta K \approx \Delta K^{\prime}$, an assumption that is justified given the relatively mild colors of solar-type stars and the fact that uncertainty in the measured magnitude difference and parallax dominate the uncertainty in absolute magnitude. The mass of each companion is found by comparing its brightness to late-type dwarfs using Dotter et al. (2008) theoretical evolutionary tracks (the Dartmouth models) and also the Delfosse et al. (2000) empirical relations that correlate $M_{K}$ with mass. Differential, apparent, and absolute magnitudes are listed in Table 2.

We find that HD $53665 \mathrm{~B}$ has a mass of $0.65 \pm 0.03 M_{\odot}$ based on photometry (Dotter et al. 2008). Its absolute magnitude in each near-infrared band is consistent with this value. Using Table 5 from Kraus \& Hillenbrand (2007), HD 53665 B has colors and brightness consistent with either a K7 or M0 dwarf. For comparison, we find a mass of $0.70 \pm 0.03 M_{\odot}$ based on the measured $M_{K^{\prime}}=4.81 \pm 0.16$ (Delfosse et al. 2000).

HD $68017 \mathrm{~B}$ has a mass of $0.16 \pm 0.02 M_{\odot}$ based on photometry (Dotter et al. 2008). Its absolute magnitude is estimated only in the $K$ band, as our unocculted $H$-band observations of the primary star from 2012 January 7 were saturated. Table 2 shows a lower limit for the $H$-band magnitude. HD $68017 \mathrm{~B}$ has a $K$-band brightness consistent with an M5-dwarf (Kraus \& Hillenbrand 2007). For comparison, we find a mass of $0.15 \pm 0.01 M_{\odot}$ based on the measured $M_{K^{\prime}}=$ $8.31 \pm 0.11$ (Delfosse et al. 2000).

HD $71881 \mathrm{~B}$ has a mass of $0.31 \pm 0.03 M_{\odot}$ based on photometry. Its absolute magnitudes in the $H$ and $K$ bands are both consistent with this value. HD $71881 \mathrm{~B}$ has colors and brightness most consistent with either an M3 or M4 dwarf (Kraus \& Hillenbrand 2007). For comparison, we find a mass of $0.29 \pm 0.02 M_{\odot}$ based on the measured $M_{K^{\prime}}=6.99 \pm 0.11$ (Delfosse et al. 2000).

\subsection{Mass Lower Limit from Dynamics}

When combined with an RV trend, a single epoch of imaging observations (single measurement of the projected physical separation) places a lower limit on the companion dynamical mass (Torres 1999; Liu et al. 2002). We have measured the instantaneous Doppler acceleration for HD 53665 and HD 71881,

\footnotetext{
11 http://www.astro.caltech.edu/jmc/2mass/v3/transformations/
} 
assuming that the RV data may be approximated as linear across the full time baseline. Using a Markov chain Monte Carlo (MCMC) analysis, we find slopes of

$$
\begin{aligned}
& (d v / d t)_{\mathrm{HD} 53665}=+5.3 \pm 0.3 \mathrm{~m} \mathrm{~s}^{-1} \mathrm{yr}^{-1} \\
& (d v / d t)_{\mathrm{HD} 71881}=-10.3 \pm 0.2 \mathrm{~m} \mathrm{~s}^{-1} \mathrm{yr}^{-1}
\end{aligned}
$$

With a projected separation of 102.9 AU (2011 February 22), HD $53665 \mathrm{~B}$ has a minimum dynamical mass of $0.77 \pm$ $0.14 M_{\odot}$. Accounting for uncertainty in the stellar parallax, measured angular separation, and RV acceleration, we adopt a minimum mass of $0.63 M_{\odot}$. This value is consistent with the mass derived from photometry provided that HD $53665 \mathrm{~B}$ has an edge-on orbit (modulo faulty assumptions regarding system coevolution, or systematic errors in the isochrone models). Likewise, HD $71881 \mathrm{~B}$ has a projected separation of 35.2 AU (2011 February 22), which corresponds to a minimum dynamical mass of $0.17 M_{\odot}$. Constraints from Doppler RV and imaging data are compared to mass estimates from photometry in Table 2.

HD $68017 \mathrm{~B}$ has a projected separation of $13.0 \pm 0.2 \mathrm{AU}$ (2011 February 22). Evaluating the local RV slope using the most recently obtained 21 data points (from 2010 September 25 to present), we find $(d v / d t)_{\mathrm{HD} 68017}=+16.3 \pm 0.9 \mathrm{~m} \mathrm{~s}^{-1} \mathrm{yr}^{-1}$, which corresponds to a minimum dynamical mass of $0.03 M_{\odot}$. However, the full RV time series shows significant curvature (change in the acceleration), enabling a more sophisticated analysis. We have performed MCMC simulations that simultaneously fit the Doppler and astrometric data using a Keplerian orbit. Our calculations account for the measured orbital motion of the companion from both imaging epochs. While the currently available data set provides insufficient information to calculate a unique orbit inclination, we find that the companion minimum mass constraint becomes $m_{\text {dyn }}>0.08 M_{\odot}(68.2 \%$ confidence).

\section{SUMMARY}

We have established a new observing program that uses precise RV measurements to identify promising targets for highcontrast imaging follow-up observations. The nearby, solartype stars HD 53665, HD 68017, and HD 71881 exhibit longterm Doppler accelerations ("trends"). We have used NIRC2 $\mathrm{AO}$ observations at Keck to directly detect the companions responsible for causing the trend in each case. Multi-epoch astrometry demonstrates that each candidate is comoving with the primary star. Relative photometry measurements suggest spectral types of K7-M0, M5, and M3-M4, respectively.

As inferred from their host star, each $\mathrm{M}$ dwarf companion is a metallicity and age benchmark object. With continued Doppler monitoring and follow-up AO observations, two out of the three companions, HD 68017 B and possibly HD $71881 \mathrm{~B}$, will also serve as mass benchmarks. HD 68017 has a projected separation of only $13.0 \pm 0.2 \mathrm{AU}$ and already shows significant RV curvature (change in the acceleration) and measurable astrometric motion, making it possible to estimate a dynamical mass in the next several years. ${ }^{12}$ Using currently available data, we calculate a lower limit to the mass of each companion. We posit that HD $53665 \mathrm{~B}$ must have a near edge-on orbit given the agreement between the mass estimate from photometry and dynamics.

\footnotetext{
12 For comparison, given the typical (high) signal-to-noise ratio of Doppler measurements for solar-type stars and direct astrometric measurements, Crepp et al. (2012) have shown it possible to calculate accurate dynamical masses for companions with semimajor axes as large as $\approx 19 \mathrm{AU}$.
}

Few mass, age, and metallicity benchmark dwarfs are currently known (Liu et al. 2007; Dupuy et al. 2010; Bowler et al. 2012a, 2012b). The goal of the TRENDS high-contrast imaging program is to discover and characterize low-mass stellar and substellar companions with physical properties determined independently from spectrophotometric measurements, in order to calibrate theoretical atmospheric models and thermal evolutionary models. Each of the companions presented is amenable to direct spectroscopy using AO-fed integral-field units, such as OSIRIS at Keck or Project 1640 at Palomar.

J.C. acknowledges support from NASA Origins grant NNX13AB03G. The data presented herein were obtained at the W. M. Keck Observatory, which is operated as a scientific partnership among the California Institute of Technology, the University of California, and the National Aeronautics and Space Administration. The Observatory was made possible by the generous financial support of the W. M. Keck Foundation.

\section{APPENDIX}

Table 3

\begin{tabular}{|c|c|c|}
\hline $\begin{array}{l}\text { HJD } \\
-2,440,000\end{array}$ & $\begin{array}{c}\mathrm{RV} \\
\left(\mathrm{m} \mathrm{s}^{-1}\right)\end{array}$ & $\begin{array}{l}\text { Uncertainty } \\
\left(\mathrm{m} \mathrm{s}^{-1}\right)\end{array}$ \\
\hline 10838.8830 & -25.83 & 5.69 \\
\hline 10861.8568 & -9.69 & 5.98 \\
\hline 11071.1199 & -16.24 & 5.84 \\
\hline 11171.9255 & -14.63 & 5.79 \\
\hline 11226.8781 & -17.12 & 5.65 \\
\hline 11544.0550 & -1.81 & 5.92 \\
\hline 11552.9643 & -20.22 & 6.06 \\
\hline 11585.9500 & -23.16 & 5.82 \\
\hline 11883.0298 & -33.55 & 5.82 \\
\hline 11973.8361 & -22.17 & 5.91 \\
\hline 12235.9597 & -16.01 & 5.60 \\
\hline 12574.0727 & 0.74 & 5.70 \\
\hline 12600.9894 & -7.03 & 5.60 \\
\hline 12653.0320 & 15.80 & 5.83 \\
\hline 12987.9295 & -13.35 & 5.77 \\
\hline 13304.1114 & 16.06 & 5.24 \\
\hline 13400.9519 & 22.45 & 5.38 \\
\hline 14024.1377 & 31.39 & 5.45 \\
\hline 15521.9975 & 32.68 & 5.38 \\
\hline 15672.8256 & 57.91 & 5.50 \\
\hline 15871.0233 & 43.77 & 5.25 \\
\hline
\end{tabular}

Radial Velocities for HD 53665

Table 4

Radial Velocities for HD 68017

\begin{tabular}{lcc}
\hline \hline $\begin{array}{l}\text { HJD } \\
-2,440,000\end{array}$ & $\begin{array}{c}\text { RV } \\
\left(\mathrm{m} \mathrm{s}^{-1}\right)\end{array}$ & $\begin{array}{c}\text { Uncertainty } \\
\left(\mathrm{m} \mathrm{s}^{-1}\right)\end{array}$ \\
\hline 10461.9269 & 50.69 & 2.36 \\
10784.1169 & 41.84 & 2.41 \\
10807.1673 & 30.26 & 2.74 \\
10837.8347 & 39.68 & 2.46 \\
10838.9684 & 35.12 & 2.53 \\
10861.8262 & 39.34 & 2.46 \\
10862.7586 & 42.87 & 2.38 \\
11171.0900 & 18.20 & 2.44 \\
11226.7940 & 31.60 & 2.51 \\
11227.9052 & 30.02 & 2.43 \\
11229.9098 & 32.27 & 2.33 \\
11551.0555 & 17.13 & 2.61 \\
11583.9188 & 15.21 & 2.46
\end{tabular}


Table 4

(Continued)

\begin{tabular}{|c|c|c|}
\hline $\begin{array}{l}\text { HJD } \\
-2,440,000\end{array}$ & $\begin{array}{c}\mathrm{RV} \\
\left(\mathrm{m} \mathrm{s}^{-1}\right)\end{array}$ & $\begin{array}{l}\text { Uncertainty } \\
\left(\mathrm{m} \mathrm{s}^{-1}\right)\end{array}$ \\
\hline 11884.1426 & 14.94 & 2.52 \\
\hline 11898.1321 & 21.48 & 2.50 \\
\hline 11899.1205 & 23.57 & 2.41 \\
\hline 11900.0979 & 18.66 & 2.33 \\
\hline 11901.1537 & 16.07 & 2.40 \\
\hline 11972.0135 & 11.99 & 2.44 \\
\hline 11972.9874 & 15.01 & 2.39 \\
\hline 11973.8854 & 16.30 & 2.45 \\
\hline 11974.8692 & 12.93 & 2.49 \\
\hline 12243.0488 & 5.48 & 2.58 \\
\hline 12573.1006 & 2.04 & 2.53 \\
\hline 12601.0207 & -2.07 & 2.44 \\
\hline 12680.9684 & -8.36 & 2.47 \\
\hline 12711.7407 & -2.32 & 2.58 \\
\hline 12988.0081 & -12.00 & 2.55 \\
\hline 13303.1395 & -6.03 & 2.78 \\
\hline 13398.8550 & -15.14 & 2.12 \\
\hline 13692.9970 & -14.80 & 2.13 \\
\hline 13693.0957 & -16.00 & 2.12 \\
\hline 13694.1285 & -17.28 & 2.13 \\
\hline 13695.1055 & -16.35 & 2.13 \\
\hline 13696.0847 & -16.56 & 2.19 \\
\hline 13697.1010 & -28.71 & 2.18 \\
\hline 13724.0212 & -17.79 & 2.15 \\
\hline 13725.0429 & -18.73 & 2.15 \\
\hline 13747.0478 & -12.93 & 2.14 \\
\hline 13747.9616 & -15.76 & 2.15 \\
\hline 13748.9262 & -16.15 & 2.37 \\
\hline 13749.8534 & -13.49 & 2.16 \\
\hline 13750.8602 & -15.83 & 2.15 \\
\hline 13751.9266 & -10.32 & 2.39 \\
\hline 13752.9635 & -14.96 & 2.14 \\
\hline 13753.9402 & -12.69 & 2.13 \\
\hline 13775.7943 & -13.93 & 2.14 \\
\hline 13776.9239 & -10.81 & 2.14 \\
\hline 14129.9829 & -16.35 & 2.14 \\
\hline 14399.1317 & -10.94 & 3.11 \\
\hline 14492.9688 & -22.14 & 2.43 \\
\hline 14547.9008 & -16.94 & 2.46 \\
\hline 14548.8355 & -11.53 & 2.53 \\
\hline 14809.9817 & -6.87 & 2.59 \\
\hline 14867.8982 & -8.70 & 2.48 \\
\hline 14927.8853 & -12.18 & 2.56 \\
\hline 14929.8538 & -2.49 & 2.48 \\
\hline 14984.7583 & -8.21 & 2.42 \\
\hline 15109.1438 & -5.35 & 3.02 \\
\hline 15134.1513 & 0.84 & 3.02 \\
\hline 15164.0166 & 1.78 & 2.44 \\
\hline 15172.1149 & -4.52 & 2.52 \\
\hline 15188.0140 & -2.65 & 2.51 \\
\hline 15189.9820 & -1.62 & 2.44 \\
\hline 15192.0018 & -0.65 & 2.49 \\
\hline 15198.9722 & -8.83 & 2.50 \\
\hline 15229.1011 & -1.10 & 2.16 \\
\hline 15229.7942 & -5.34 & 2.17 \\
\hline 15231.8098 & 2.19 & 2.17 \\
\hline 15251.9199 & 0.16 & 2.17 \\
\hline 15255.7629 & -5.62 & 2.15 \\
\hline 15260.7790 & -6.72 & 2.16 \\
\hline 15289.7242 & 2.54 & 2.50 \\
\hline 15311.7447 & 3.50 & 2.49 \\
\hline 15344.7419 & -6.89 & 2.69 \\
\hline 15465.1494 & 5.98 & 2.36 \\
\hline 15468.1055 & 4.81 & 2.36 \\
\hline 15469.1528 & 3.65 & 2.37 \\
\hline
\end{tabular}

Table 4

(Continued)

\begin{tabular}{lcc}
\hline \hline $\begin{array}{l}\text { HJD } \\
-2,440,000\end{array}$ & $\begin{array}{c}\text { RV } \\
\left(\mathrm{m} \mathrm{s}^{-1}\right)\end{array}$ & $\begin{array}{c}\text { Uncertainty } \\
\left(\mathrm{m} \mathrm{s}^{-1}\right)\end{array}$ \\
\hline 15470.1502 & 9.43 & 2.36 \\
15487.1576 & 5.54 & 2.35 \\
15490.1555 & 8.16 & 2.41 \\
15491.1524 & 8.56 & 2.35 \\
15501.1589 & 9.99 & 2.38 \\
15522.0236 & 2.12 & 2.27 \\
15543.1041 & 3.73 & 2.16 \\
15605.9635 & 16.44 & 2.19 \\
15634.8186 & 8.89 & 2.16 \\
15671.7972 & 14.31 & 2.13 \\
15697.7307 & 12.49 & 2.17 \\
15698.7813 & 11.57 & 2.18 \\
15843.0775 & 24.08 & 2.38 \\
15880.1365 & 28.22 & 2.93 \\
15904.1782 & 22.53 & 2.58 \\
15960.9100 & 33.29 & 2.55 \\
15999.7837 & 27.41 & 2.52 \\
16073.7601 & 29.82 & 2.49 \\
\hline
\end{tabular}

Table 5

Radial Velocities for HD 71881

\begin{tabular}{lrc}
\hline \hline $\begin{array}{l}\text { HJD } \\
-2,440,000\end{array}$ & $\begin{array}{c}\mathrm{RV} \\
\left(\mathrm{m} \mathrm{s}^{-1}\right)\end{array}$ & $\begin{array}{c}\text { Uncertainty } \\
\left(\mathrm{m} \mathrm{s}^{-1}\right)\end{array}$ \\
\hline 10807.1721 & 112.06 & 2.56 \\
10837.9895 & 106.74 & 2.37 \\
10862.7614 & 109.55 & 2.46 \\
10862.8804 & 108.43 & 2.37 \\
11171.0933 & 97.73 & 2.35 \\
11227.9372 & 98.27 & 2.32 \\
11552.0265 & 79.55 & 2.42 \\
11900.1047 & 76.05 & 2.33 \\
12003.9078 & 69.54 & 2.47 \\
12007.8729 & 66.09 & 2.39 \\
12062.7530 & 69.57 & 2.47 \\
12064.7717 & 71.06 & 2.55 \\
12236.0510 & 56.62 & 2.54 \\
12573.1159 & 51.72 & 2.40 \\
12712.8234 & 48.80 & 2.50 \\
12988.0181 & 45.16 & 2.43 \\
13303.1333 & 50.16 & 3.91 \\
13425.8843 & 25.04 & 4.67 \\
15556.1133 & -8.53 & 4.41 \\
15871.0606 & -34.85 & 4.41 \\
16018.8872 & -40.97 & 4.52 \\
\hline & &
\end{tabular}

\section{REFERENCES}

Barman, T. S., Macintosh, B., Konopacky, Q. M., \& Marois, C. 2011, ApJ, 733,65

Biller, B. A., Close, L. M., Masciadri, E., et al. 2007, ApJS, 173, 143

Biller, B. A., Liu, M. C., Wahhaj, Z., et al. 2010, ApJ, 720, L82

Boden, A. F., Torres, G., \& Latham, D. W. 2006, ApJ, 644, 1193

Bowler, B. P., Liu, M. C., Dupuy, T. J., \& Cushing, M. C. 2010, ApJ, 723, 850

Bowler, B. P., Liu, M. C., Shkolnik, E. L., \& Tamura, M. 2012a, ApJ, 756, 69

Bowler, B. P., Liu, M. C., Shkolnik, E. L., et al. 2012b, ApJ, 753, 142

Burrows, A., Marley, M., Hubbard, W. B., et al. 1997, ApJ, 491, 856

Butler, R. P., Marcy, G. W., Williams, E., et al. 1996, PASP, 108, 500

Carpenter, J. M. 2001, AJ, 121, 2851

Crepp, J., Serabyn, E., Carson, J., Ge, J., \& Kravchenko, I. 2010, ApJ, 715, 1533

Crepp, J. R., Johnson, J. A., Fischer, D. A., et al. 2012, ApJ, 751, 97

Crepp, J. R., Pueyo, L., Brenner, D., et al. 2011, ApJ, 729, 132

Delfosse, X., Forveille, T., Ségransan, D., et al. 2000, A\&A, 364, 217

Dotter, A., Chaboyer, B., Jevremović, D., et al. 2008, ApJS, 178, 89 
Dupuy, T. J., Liu, M. C., Bowler, B. P., et al. 2010, ApJ, 721, 1725

Dupuy, T. J., Liu, M. C., \& Ireland, M. J. 2009, ApJ, 692, 729

Ehrenreich, D., Lagrange, A., Montagnier, G., et al. 2010, A\&A, 523, A73

Ghez, A. M., Salim, S., Weinberg, N. N., et al. 2008, ApJ, 689, 1044

Guyon, O., Pluzhnik, E. A., Kuchner, M. J., Collins, B., \& Ridgway, S. T. 2006, ApJS, 167, 81

Hinkley, S., Oppenheimer, B. R., Zimmerman, N., et al. 2011, PASP, 123,74

Howard, A. W., Johnson, J. A., Marcy, G. W., et al. 2010, ApJ, 721, 1467

Janson, M., Carson, J., Thalmann, C., et al. 2011, ApJ, 728, 85

Johnson, J. A., \& Apps, K. 2009, ApJ, 699, 933

Kraus, A. L., \& Hillenbrand, L. A. 2007, AJ, 134, 2340

Lafrenière, D., Doyon, R., Marois, C., et al. 2007, ApJ, 670, 1367

Leconte, J., Soummer, R., Hinkley, S., et al. 2010, ApJ, 716, 1551

Liu, M. C., Fischer, D. A., Graham, J. R., et al. 2002, ApJ, 571, 519

Liu, M. C., Leggett, S. K., \& Chiu, K. 2007, ApJ, 660, 1507

Mamajek, E. E., \& Hillenbrand, L. A. 2008, ApJ, 687, 1264

Marcy, G. W., \& Butler, R. P. 1992, PASP, 104, 270

Marois, C., Lafrenière, D., Doyon, R., Macintosh, B., \& Nadeau, D. 2006, ApJ, 641,556
Masciadri, E., Mundt, R., Henning, T., Alvarez, C., \& Barrado y Navascués, D. 2005, ApJ, 625, 1004

Metchev, S. A., \& Hillenbrand, L. A. 2009, ApJS, 181, 62

Nielsen, E. L., \& Close, L. M. 2010, ApJ, 717, 878

Pueyo, L., Crepp, J. R., Vasisht, G., et al. 2012, ApJS, 199, 6

Rodigas, T. J., Males, J. R., Hinz, P. M., Mamajek, E. E., \& Knox, R. P. 2011, ApJ, 732, 10

Skemer, A. J., Hinz, P. M., Esposito, S., et al. 2012, ApJ, 753, 14

Skrutskie, M. F., Cutri, R. M., Stiening, R., et al. 2006, AJ, 131, 1163

Skrutskie, M. F., Jones, T., Hinz, P., et al. 2010, Proc. SPIE, 7735, 118

Soummer, R., Pueyo, L., \& Larkin, J. 2012, ApJ, 755, L28

Stevenson, D. J. 1991, ARA\&A, 29, 163

Tokunaga, A. T., Simons, D. A., \& Vacca, W. D. 2002, PASP, 114, 180

Torres, G. 1999, PASP, 111, 169

Valenti, J. A., \& Fischer, D. A. 2005, ApJS, 159, 141

van Leeuwen, F. 2007, A\&A, 474, 653

Vigan, A., Patience, J., Marois, C., et al. 2012, A\&A, 544, A9

Vogt, S. S., Allen, S. L., Bigelow, B. C., et al. 1994, Proc. SPIE, 2198, 362

Wizinowich, P., Acton, D. S., Shelton, C., et al. 2000, PASP, 112, 315 